\title{
THE SUBCONTINUA OF A DENDRON FORM A HILBERT CUBE FACTOR ${ }^{1}$
}

\author{
JAMES E. WEST ${ }^{2}$
}

\begin{abstract}
The title statement is proved, and it is shown further that the subcontinua of a dendron actually form a Hilbert cube when (and only when) the branch points of the dendron are dense. Along the way, it is established that whenever a Hilbert cube manifold is compactified into a Hilbert cube factor by the addition of another Hilbert cube factor having Property $\mathrm{Z}$ in the compactification, then the resulting space is actually a Hilbert cube.
\end{abstract}

It is known ([1], [14]) that the Cartesian product of any dendron with the Hilbert cube is homeomorphic to the Hilbert cube. In this paper, the hyperspace $C(D)$ of all nonvoid subcontinua of a dendron $D$ is investigated and found to have the same property. In fact, it is shown that if the branch points of $D$ are dense, then $C(D)$ is a Hilbert cube.

The method used presents in simple form the techniques employed elsewhere by the author and R. M. Schori ([11], [12]) to show that the hyperspace of all nonvoid, closed subsets of the unit interval is a Hilbert cube, and Theorem 1 is applied crucially in the extension of that result to finite, connected graphs in [13].

The space $X$ will be termed a Hilbert cube factor if for some space $Y$, $X \times Y$ is homeomorphic to the Hilbert cube, $Q$. It is easy to see that this condition holds if and only if $X \times Q$ is a Hilbert cube. (See [15, Lemma 2].)

A dendron $D$ is a uniquely arcwise connected Peano continuum. The branch points of $D$ are those points which separate it into more than two

Received by the editors August 2, 1971.

AMS 1970 subject classifications. Primary 54B20, 54D35, 54F50, 57A20; Secondary 54B10, 54B25.

Key words and phrases. Hyperspace of subcontinua, dendron, Hilbert cube, Hilbert cube factor, Hilbert cube manifold, compactification.

${ }_{1}$ This material was presented by the author to the Conference on Infinite-Dimensional Topology at the Mathematisches Forschungsinstitut in Oberwolfach, West Germany, in September, 1970.

2 The research was partially done while the author was supported by NSF grant GP-16862 and completed while the author was supported by a travel grant from the Mathematisches Forschungsinstitut. Theorem 1 was born in a conversation with T. A. Chapman at the International Congress of Mathematicians in Nice, France, during September, 1970.

(c) American Mathematical Society 1973 
components. Furthermore, each dendron $D$ may be written as the closure in the plane of a countable union of $\operatorname{arcs} \alpha_{i}$, of lengths converging to zero, such that

$$
\alpha_{i} \cap\left(T_{i-1}=\bigcup_{j=1}^{i-1} \alpha_{j}\right)=a_{i},
$$

is an endpoint of $\alpha_{i}$ [16]. This, together with the unique arcwise connectivity, allows the dendron to be written as the $\operatorname{inv} \lim \left\{T_{i}, r_{i}\right\}$, where $r_{i}: T_{i} \rightarrow T_{i-1}$ is defined by retracting $\alpha_{i}$ to $a_{i}$. (The $r_{i}$ 's extend to retractions of $D$.) Finally, a dendron always admits a convex metric $\rho$, i.e., one for which there always exists a point halfway between any two given points. The metric $\rho$ may always be chosen so that $b$ separates $a$ from $c$ in $D$ if and only if the points $a, b$, and $c$ are distinct and $\rho(a, c)=\rho(a, b)+\rho(b, c)$. (See [14] for a simple construction of such a metric by embedding in the Hilbert cube.)

We are concerned with the hyperspace $C(D)$ of all (nonvoid) subcontinua of $D$ under the topology generated by the Hausdorff metric $d_{H}$, which is defined by the condition that $d_{H}\left(K_{1}, K_{2}\right)<\varepsilon$ if and only if each point of $K_{1}$ is within $\varepsilon$ of some point of $K_{2}$ and vice versa. The inverse limit expression inv $\lim \left\{T_{i}, r_{i}\right\}$ of $D$ given above induces one inv $\lim \left\{C\left(T_{i}\right), \bar{r}_{i}\right\}$ of $C(D)$ when $\bar{r}_{i}$ is the map $C\left(T_{i}\right) \rightarrow C\left(T_{i-1}\right)$ induced by $r_{i}$. We shall show that this expression guarantees that $C(D)$ is a Hilbert cube factor, but first it is necessary to introduce some notation.

A closed subset $A$ of a separable, metric ANR $X$ has Property $\mathrm{Z}$ in $X$ if and only if for every open set $U$ of $X$ the inclusion map $U \backslash A \rightarrow U$ is a homotopy equivalence. (This is the appropriate generalization to ANR's of R. D. Anderson's definition [2] of Property Z in infinite-dimensional manifolds. See, for example, the article by Eells and Kuiper [9], in which it is shown that $A$ has Property $\mathrm{Z}$ in $X$ if for every $a \in A$ there are arbitrarily small open neighborhoods $U$ of $a$ in $X$ with the property that each map of a sphere into $U$ may be deformed in $U$ to a map into $U \backslash A$, and each map $f:\left(B^{n}, S^{n-1}\right) \rightarrow(U, U \backslash A)$ is homotopic in $U$ to a map $g: B^{n} \rightarrow U \backslash A$ by a homotopy which is constant on $S^{n-1} \times I$. See also [4]. This condition is guaranteed whenever there is a homotopy $H: X \times I \rightarrow X$ with $H(x, 0)=x$ for each $x \in X$ which has the property that $H(X \times(0,1]) \cap$ $A=\varnothing$.) The importance of Property $\mathrm{Z}$ in the Hilbert cube is expressed by Anderson's homogeneity theorem [2, Theorem 10.1]: Each homeomorphism between two subsets of the Hilbert cube with Property $\mathrm{Z}$ extends to a homeomorphism of the Hilbert cube. This theorem will be used immediately to establish Theorem 1 below, which gives a sufficient condition for the compactification of a Hilbert cube manifold to be a Hilbert cube. 
THEOREM 1. Let $X$ be a compactification of a Hilbert cube manifold $M$ such that both $X$ and the remainder, $A=X \backslash M$, are Hilbert cube factors. If $A$ has Property $\mathrm{Z}$ in $X$, then $X$ is a Hilbert cube.

Proof. The pair $(X, A)$ is a pair of Hilbert cube factors, so $(X \times Q$, $A \times Q)$ is a pair of Hilbert cubes. Moreover, $A \times Q$ has Property $\mathrm{Z}$ in $X \times Q$, as is easily seen. The homogeneity theorem then allows a homeomorphism of pairs $(X \times Q, A \times Q) \rightarrow(A \times Q \times I, A \times Q \times\{1\})$ extending the natural homeomorphism on the second entries. Thus, the space $Y$ obtained from $X \times Q$ by simultaneously identifying $\{a\} \times Q$ to a point $a$ for each $a$ in $A$ is homeomorphic to the result, $Z$, of identifying each $\{a\} \times Q \times\{1\}$ to a point in $A \times Q \times I$. However, $Z$ is just the product of $A$ with the cone $C(Q)$ of $Q$. Note that $C(Q)$ is a Hilbert cube: One way of seeing this is to observe that $Q$, hence $C(Q)$, may be embedded in Hilbert space as a compact, convex, infinite-dimensional set, so the theorem of O. H. Keller, [10] that all such are Hilbert cubes applies. Since $C(Q)$ is a Hilbert cube and $A$ is a Hilbert cube factor, $Z$ is also a Hilbert cube, and so is $Y$. There remains only to show that $Y$ is homeomorphic to $X$, which is done as follows: $Y \backslash A$ is homeomorphic to $X \backslash A$ by [3], because it is homeomorphic to $X \times Q \backslash A \times Q=M \times Q$ and each Hilbert cube manifold is homeomorphic to its product with a Hilbert cube. Also, $Y \mid A$, the result of identifying $A$ to a point, is a Hilbert cube because $Y \mid A$ is homeomorphic to $Z / A=$ $C(A \times Q)$ which is homeomorphic to a Hilbert cube. Hence, $Y \backslash A$, and therefore $M$, embeds in $Q$ as an open subset. Now, the proof of the theorem in the addendum of [3] shows that for any open cover $\mathscr{U}$ of $M$ there is a homeomorphism $h: M \times Q \rightarrow M$ which is a $\mathscr{U}$-close to the projection $p: M \times Q \rightarrow M$, i.e., the cover $\{\{p(x), h(x)\} \mid x \in M \times Q\}$ refines $\mathscr{U}$. The proper choice of $\mathscr{U}$ yields a homeomorphism of $Y \backslash A$ onto $M$ extending to a homeomorphism of $Y$ onto $X$ which is the identity on $A$. Since $Y$ is a Hilbert cube, so is $X$.

The following is the main theorem of this note.

THEOREM 2. The subcontinua $C(D)$ of a dendron $D$ form a Hilbert cube factor which is a Hilbert cube if (and only if) the branch points of $D$ are dense.

Proof. Write $D=\operatorname{inv} \lim \left\{T_{i}, r_{i}\right\}$ and for each $i>1$ let $b_{i}$ be the endpoint of the arc $\alpha_{i}=\operatorname{cl}\left(T_{i} \backslash T_{i-1}\right)$ which does not lie in $T_{i-1}$. Now consider

$$
r_{i}^{\prime}=\bar{r}_{i} \mid C_{b_{i}}\left(T_{i}\right): C_{b_{i}}\left(T_{i}\right) \rightarrow C\left(T_{i-1}\right)
$$

where $C_{b_{i}}\left(T_{i}\right)$ denotes those members of $C\left(T_{i}\right)$ containing $b_{i}$. Now let $M_{r_{i}}$ be the mapping cylinder of $r_{i}^{\prime}$, that is,

$$
M_{r_{i^{\prime}}}=\left[C_{b_{i}}\left(T_{i}\right) \times I \cup C\left(T_{i-1}\right)\right] / \Xi,
$$


where $\Xi$ is the upper-semicontinuous decomposition with nondegenerate elements all sets of the form $\{K\} \cup\left(r_{i}^{\prime-1}(K) \times\{0\}\right)$ where $K$ is in $C_{a_{i}}\left(T_{i-1}\right)$.

There is a homeomorphism $g_{i}$ of $C\left(T_{i}\right)$ onto $M_{r_{i}}$, for which the following diagram commutes, $c_{i}$ being the collapse of $M_{r_{i}^{\prime}}$ to $C\left(T_{i-1}\right)$ given by $[K, t] \rightarrow r_{i}^{\prime}(K)$ if $K$ is in $C_{b_{i}}\left(T_{i}\right)$ and $[K] \rightarrow K$ if $K$ is in $C\left(T_{i-1}\right)$.

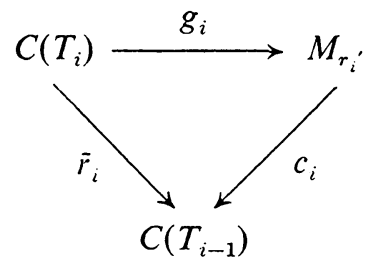

This $g_{i}$ may be defined by parameterizing $\alpha_{i}$ with $[0,1]$ so that $a_{i} \leftrightarrow 0$ and $b_{i} \leftrightarrow 1$ and then letting

$$
\begin{aligned}
g_{i}(K) & =[K], & & \text { if } K \cap \alpha_{i}=\varnothing, \\
& =\left[K \cup \alpha_{i}, d\right], & & \text { if } a_{i} \in K, \\
& =[(1 / d) K, d], & & \text { if } K \subset \alpha_{i} \mid\left\{a_{i}\right\},
\end{aligned}
$$

where $d=\sup \left(K \cap \alpha_{i}\right)$ if $K \cap \alpha_{i} \neq \varnothing$.

Both $C\left(T_{i}\right)$ and $C_{\iota_{i}}\left(T_{i}\right)$ are polyhedra by [6] and [8] (see also [7] for a definitive analysis), and they are easily seen to be contractible because $T_{i}$ is contractible to $b_{i}$. Therefore, by [14] they are Hilbert cube factors, so by [15],

$$
c_{i} \times \mathrm{id}: M_{r^{\prime}} \times Q \rightarrow C\left(T_{i-1}\right) \times Q
$$

is a uniform limit of homeomorphisms. The commutative diagram above shows that

$$
\bar{r}_{i} \times \mathrm{id}: C\left(T_{i}\right) \times Q \rightarrow C\left(T_{i-1}\right) \times Q
$$

is also a uniform limit of homeomorphisms. Thus,

$$
\text { inv } \lim \left\{C\left(T_{i}\right) \times Q, \bar{r}_{i} \times \mathrm{id}\right\},
$$

being an inverse limit of Hilbert cubes and uniform limits of homeomorphisms, is a Hilbert cube by [5]. However, inv $\lim \left\{C\left(T_{i}\right) \times Q, \bar{r}_{i} \times\right.$ id $\}$ is easily seen to be homeomorphic to inv $\lim \left\{C\left(T_{i}\right), \bar{r}_{i}\right\} \times Q=C(D) \times Q$, so $C(D)$ is a Hilbert cube factor.

If now, the branch points of $D$ are not dense, it is immediate that $C(D)$ contains an open 2-cell, namely, those nondegenerate members which lie entirely within some open arc which is an open subset of $D$. Therefore, in this case $C(D)$ is not itself a Hilbert cube.

On the other hand, if the branch points of $D$ are dense, Theorem 1 may be employed to show $C(D)$ a Hilbert cube by identifying $D$ with the set 
of all degenerate subcontinua of itself and showing that (1) it has Property $\mathrm{Z}$ in $C(D)$, and (2) $C(D) \backslash D$ is a Hilbert cube manifold. ( $D$ itself is a Hilbert cube factor as may be seen by using the proof already given for $C(D)$ or by citing [14]. The fact was originally proven by $\mathrm{R}$. D. Anderson [1], but his proof was never published.)

It is easy to show that $D$ has Property $\mathrm{Z}$ in $C(D)$, because, using the metric $\rho$ selected at the outset, the homotopy $H: C(D) \times I \rightarrow C(D)$ sending $(K, t)$ to the closed $t$-neighborhood of $K$ in $D$ satisfies the homotopy condition mentioned parenthetically which guarantees Property $Z$. There remains, then, only to verify that $C(D) \backslash D$ is a Hilbert cube manifold. Again using the metric $\rho$, it is easy to see that for any nondegenerate subcontinuum $K$ of $D$ there are an $\varepsilon>0$ and two other nondegenerate subcontinua $K_{-}$and $K_{+}$of $D$ with the property that the closed $\varepsilon$-neighborhood $N$ of $K$ in $C(D)$ is the set of all members of $C(D)$ containing $K$ yet lying in $K_{+}$. Setting $H=K_{+} / K_{-}$, it is easy to see that the map $C\left(K_{+}\right) \rightarrow C(H)$ induced from $K_{+} \rightarrow K_{+} / K_{-}$carries $N$ homeomorphically to $C_{*}(H)$, where $*=K_{-} / K_{-}$. Also, because the branch points of $D$ are dense, the branch points of $K_{+}$lying in $K_{-}$are dense in $K_{-}$, and thus $*$ separates $H$ into infinitely many components with closures $H_{i}, i=1,2, \cdots$. Now, the intersection maps $\bigcap H_{i}: C_{*}(H) \rightarrow C_{*}\left(H_{i}\right)$ are continuous, and their product $\bigcap: C_{*}(H) \rightarrow \prod_{i=1}^{\infty} C_{*}\left(H_{i}\right)$ is a homeomorphism. Furthermore, the proof that $C(D)$ is a Hilbert cube factor will also apply without modification to show that each $C_{*}\left(H_{i}\right)$ is one also. Therefore, $N$ is homeomorphic to a countably infinite product of nondegenerate Hilbert cube factors and by [14] is itself a Hilbert cube. This shows that $C(D) \backslash D$ is a Hilbert cube manifold, so Theorem 1 applies to show that $C(D)$ is a Hilbert cube.

\section{REFERENCES}

1. R. D. Anderson, The Hilbert cube as a product of dendrons, Notices Amer. Math. Soc. 11 (1964), 572. Abstract \#614-649.

2. - On topological infinite deficiency, Michigan Math. J. 14 (1967), 365-383. MR 35 \#4893.

3. R. D. Anderson and R. M. Schori, Factors of infinite-dimensional manifolds, Trans. Amer. Math. Soc. 142 (1969), 315-330. MR 39 \#7631.

4. R. D. Anderson, D. W. Henderson and J. E. West, Negligible subsets of infinitedimensional manifolds, Compositio Math. 21 (1969), 143-150. MR 39 \#7630.

5. M. Brown, Some applications of an approximation theorem for inverse limits, Proc. Amer. Math. Soc. 11 (1960), 478-483. MR 22 \#5959.

6. R. Duda, On the hyperspace of subcontinua of a finite graph. I, Fund. Math. 62 (1968), 265-286. MR 38 \#5175a.

7. - On the hyperspace of subcontinua of a finite graph. II, Fund. Math. 63 (1968), 225-255. MR 38 \#5175b.

8. - Correction to the paper "On the hyperspace of subcontinua of a finite graph. I", Fund. Math. 69 (1970), 207-211. MR 42 \#8453. 
9. J. Eells, Jr. and N. H. Kuiper, Homotopy negligible subsets, Composito Math. 21 (1969), 155-161. MR 40 \#6546.

10. O. H. Keller, Die Homöomorphie der kompakten konvexen Mengen im Hilbertschen Raum, Math. Ann. 105 (1931), 748-758.

11. R. M. Schori and J. E. West, $2^{I}$ is homeomorphic to the Hilbert cube, Bull. Amer. Math. Soc. 78 (1972), 402-406.

12. - The hyperspace of the unit interval (to appear).

13. - Hyperspaces of finite graphs (to appear).

14. J. E. West, Infinite products which are Hilbert cubes, Trans. Amer. Math. Soc. 150 (1970), 1-25. MR 42 \#1055.

15. - Mapping cylinders of Hilbert cube factors, General Topology and Appl. 1 (1971), 111-125.

16. G. T. Whyburn, Analytic topology, Amer. Math. Soc. Colloq. Publ., vol. 28, Amer. Math. Soc., Providence, R.I., 1942. MR 4, 86.

Department of Mathematics, Cornell University, Ithaca, New York 14850 